\section{Prevalência de síndrome metabólica em adolescentes: uma revisão sistemática}

\author{
Prevalence of metabolic syndrome in adolescents: \\ a systematic review
}

\author{
1 Departamento de Educação \\ Física, Universidade Estadual \\ de Londrina. Londrina \\ Brasil. \\ 2 Departamento de Farmácia \\ e Farmacologia, Universidade \\ Estadual de Maringá, \\ Maringá, Brasil. \\ Correspondência \\ A. C. F. Moraes \\ Grupo de Estudo e Pesquisa \\ em Metabolismo, Nutrição e \\ Exercício/Grupo de Estudo e \\ Pesquisa em Epidemiologia \\ da Atividade Física, Centro \\ de Educação Física e Esporte, \\ Departamento de Educação \\ Física, Universidade Estadual \\ de Londrina. \\ Rua Ivaí 952, apto. 64, \\ Maringá, $P R$ \\ 87005-270, Brasil. \\ moraes82@yahoo.com.br
}

\section{Abstract}

The present study aimed to review the literature on the prevalence of metabolic syndrome and its components in adolescents (10-19 years old). The search was conducted in online databases (MEDLINE and SciELO), references from retrieved articles, and contacts with authors. Only original articles using either the criteria of the World Health Organization (WHO) or the National Cholesterol Education Program's Adult Treatment Panel III (NCEP ATP-III) were considered. Sixteen studies met the inclusion criteria. Eight studies were undertaken in low to medium-income countries, but none of them in Brazil. Prevalence of metabolic syndrome in studies using the NCEP-ATP III criteria ranged from $4.2 \%$ to $15.4 \%$. Prevalence in studies using the WHO criteria was slightly higher: $4.5 \%$ to $38.7 \%$. High triglyceride level was the most frequent component of the metabolic syndrome, with prevalence ranging from $4.9 \%$ to $75.0 \%$, while high blood glucose showed the lowest prevalence. In conclusion, although there are few available studies, metabolic syndrome in adolescents is a highly prevalent health issue. The exact prevalence of metabolic syndrome in Brazilian adolescents is unknown.

Adolescent; Metabolic X Syndrome; Obesity
Augusto César Ferreira de Moraes 1,2 Camila Sanchez Fulaz 2

Edna Regina Netto-Oliveira 2

Felipe Fossati Reichert 1

\section{Introdução}

A síndrome metabólica é um transtorno representado por um conjunto de fatores de risco cardiovasculares, tais como hipertensão arterial, deposição central de gordura, dislipidemia (LDLcolesterol e triglicérides elevados e, HDL-colesterol reduzido) e resistência à insulina ${ }^{1}$. Essa síndrome foi identificada pela primeira vez em 1922 e tem sido descrita por diferentes terminologias como quarteto mortal, síndrome X, síndrome plurimetabólica e síndrome de resistência à insulina 2 .

A ocorrência da síndrome metabólica em crianças e adolescentes está associada a risco aumentado de doenças cardiovasculares, problemas psicossociais, metabolismo anormal de glicose, distúrbios hepáticos e gastrintestinais, apnéia do sono, complicações ortopédicas $3 \mathrm{e}$ distúrbios no desenvolvimento motor 4 . Além disso, a síndrome metabólica adquirida na juventude, assim como seus riscos à saúde, tende a persistir na idade adulta ${ }^{3}$

Os critérios diagnósticos da síndrome metabólica mais utilizados são os da Organização Mundial da Saúde (OMS) 5 e os do National Cholesterol Education Program's Adult Treatment Panel III (NCEP-ATP III) 6, e algumas diferenças entre eles são observadas. A definição da OMS requer a avaliação da resistência à insulina ou do distúrbio do metabolismo da glicose. Por outro lado, a definição da NCEP-ATP III não 
exige a mensuração de resistência à insulina, facilitando sua utilização em estudos epidemiológicos 7 .

Segundo o NCEP-ATP III ${ }^{6}$, a síndrome metabólica representa a combinação de três ou mais dos seguintes componentes: deposição central de gordura, triglicérides elevados, baixos níveis de HDL colesterol, pressão arterial elevada e glicemia em jejum elevada. Pela simplicidade e praticidade é a definição recomendada pela Sociedade Brasileira de Cardiologia 7.

Os critérios da OMS e NCEP-ATP III foram formulados para adultos, e não há uma definição amplamente aceita para síndrome metabólica na adolescência ${ }^{8}$. Cook et al. 9 adaptaram os critérios do NCEP-ATP III e propuseram como definição de síndrome metabólica em populações pediátricas a presença de três ou mais dos seguintes critérios: obesidade abdominal $\geq$ percentil 8010 , glicemia de jejum $\geq 110 \mathrm{mg} / \mathrm{dL}$, triglicérideos $\geq 100 \mathrm{mg} / \mathrm{dL}$, HDL-colesterol < $40 \mathrm{mg} / \mathrm{dL}$ e pressão arterial $\geq$ percentil 90 ajustados para idade, sexo e percentil de altura 11 . Contudo, não há padronização sobre a medida de circunferência abdominal em adolescentes. Taylor et al. ${ }^{10}$ padronizaram pontos de corte por faixa etária e por sexo e consideraram elevada a medida acima do percentil 80 , entretanto, algumas pesquisas 12,13 utilizam diferentes tabelas de referência para a obesidade abdominal que a desenvolvida por Taylor et al. 10 .

Até o presente, nenhuma revisão sistemática foi conduzida com o objetivo de verificar a prevalência da síndrome metabólica em adolescentes. Sendo assim, o objetivo desta revisão sistemática foi revisar e analisar a literatura acerca da prevalência da síndrome metabólica e seus componentes em adolescentes entre 10 e 19 anos de idade. Uma descrição dos estudos em relação ao ano de publicação, país onde foi realizado, número de participantes e faixa etária incluída também é apresentada.

\section{Métodos}

Realizou-se busca nas bases de dados eletrônicas MEDLINE e SciELO, considerando o período inicial dos bancos de dados até agosto de 2007. Os seguintes descritores foram utilizados: (1) metabolic syndrome; (2) metabolic syndrome X; (3) plurimetabolic syndrome; (4) central obesity; (5) insulin resistance; (6) syndrome $X$; (7) adolescence; (8) youth; (9) teen; (10) teenager; (11) crosssectional studies; e (12) survey. Além disso, as referências dos artigos encontrados foram revisadas e também foi realizado contato com autores a fim de localizar outros estudos relevantes.
Os critérios de inclusão foram: (1) apenas estudos originais; (2) amostra que incluíssem adolescentes (10-19 anos); (3); delineamento transversal; e (4) critério do NCEP-ATP III ou OMS para diagnósticos da síndrome metabólica. Esses critérios foram utilizados para aumentar a comparabilidade dos achados entre os estudos.

As prevalências dos desfechos e seus respectivos intervalos de 95\% de confiança (IC95\%) são apresentados. O IC95\% foi extraído diretamente dos artigos, quando disponível 9,14,15,16,17, ou calculado no programa estatístico Stata 8.0 (Stata Corp., College Station, Estados Unidos) utilizando o comando "cii" (IC95\% exato para distribuições binomiais).

\section{Resultados}

Vinte e seis estudos foram inicialmente localizados e, destes, 16 atendiam aos critérios de inclusão estabelecidos para a presente revisão e dez estudos foram excluídos porque não definiram a ocorrência da síndrome metabólica pelos critérios do NCEP-ATP III ou OMS. A Figura 1 apresenta os países com estudos sobre prevalência de síndrome metabólica em adolescentes de acordo com a localização geográfica. Oito estudos foram desenvolvidos em países de renda baixa ou média (China 12, Coréia do Sul 13, México 14,18, Índia 15, Irã 16 e Turquia 19,20). Entre os restantes, sete estudos foram realizados nos Estados Unidos 9,17,21,22,23,24,25 e um na Itália 26 . Nenhum estudo foi realizado no Brasil.

A Tabela 1 apresenta uma descrição dos 16 trabalhos em relação ao primeiro autor, país onde foi realizado o estudo, ano de publicação, periódico publicado, número de participantes total no estudo, número de adolescentes, faixa etária e proporção de moças no estudo. Todos os artigos foram publicados após o ano de 2002 e em periódicos Qualis A Internacional, de acordo com a classificação da Coordenação de Aperfeiçoamento de Pessoal de Nível Superior (CAPES) no biênio 2004-2006. Seis artigos incluíram, além de adolescentes, crianças (até 10 anos) e um incluiu adultos (20 anos ou mais). Todos os estudos incluíram moças e rapazes.

A Tabela 2 apresenta o critério para definição da síndrome metabólica utilizado nos estudos, a prevalência e seus respectivos IC95\%, de cada um dos fatores de risco para o desenvolvimento da síndrome e a prevalência e os IC95\% da síndrome metabólica. Doze estudos utilizaram os procedimentos metodológicos do NCEP-ATP III, um utilizou somente os critérios da OMS e outros quatro estudos utilizaram, concomitantemente, os dois critérios. Porém, apenas três estudos 15,22,23 


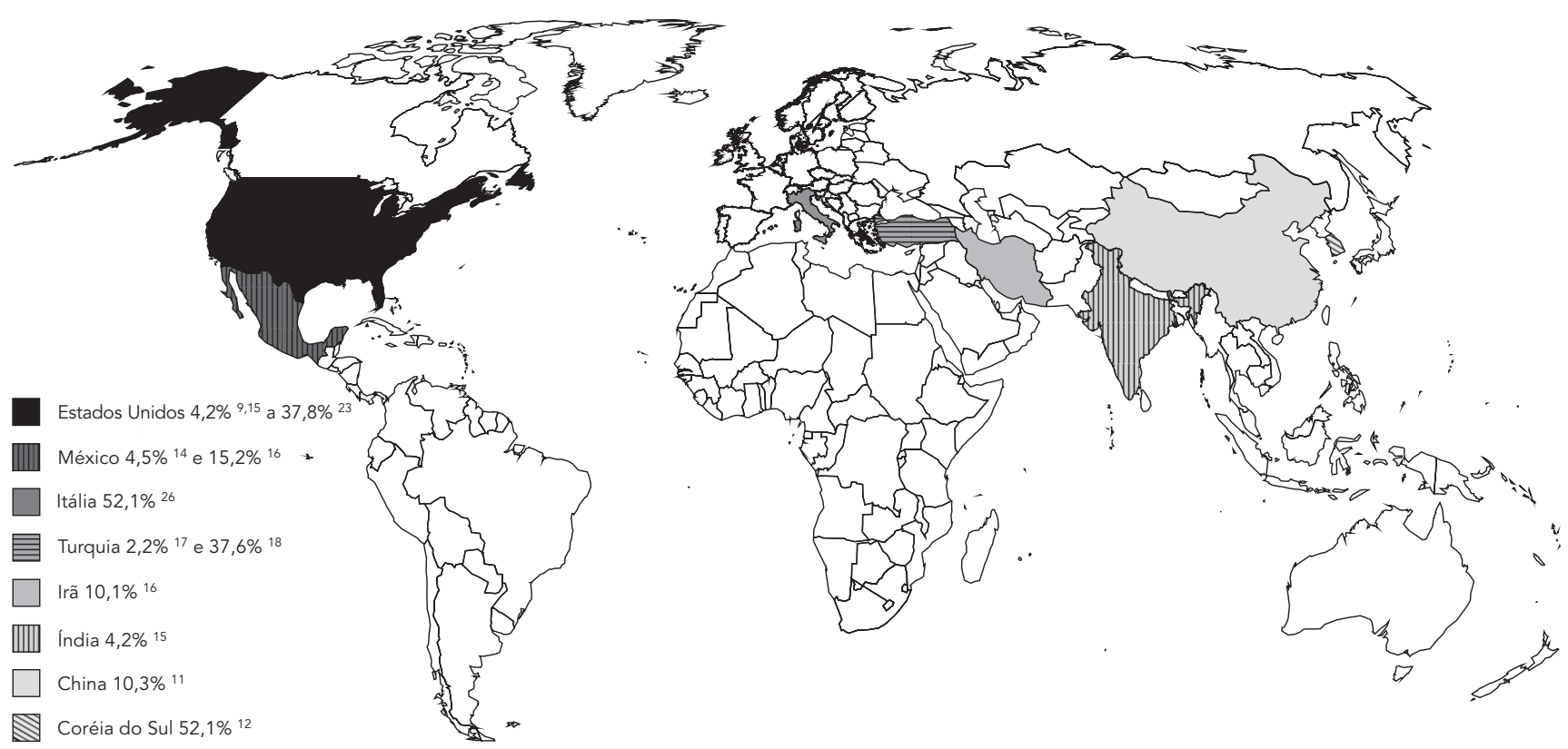

Tabela 1

Análise descritiva dos estudos revisados.

\begin{tabular}{|c|c|c|c|c|c|c|c|}
\hline Referência & País & Ano & Periódico * & $\begin{array}{c}\text { n total do } \\
\text { estudo }\end{array}$ & $\begin{array}{c}\text { Adolescentes } \\
\text { (n) }\end{array}$ & $\begin{array}{c}\text { Faixa etária } \\
\text { (anos) }\end{array}$ & $\begin{array}{r}\text { Proporção } \\
\text { de moças }\end{array}$ \\
\hline Cook et al. 9 & Estados Unidos & 2003 & Arch Pediatr Adolesc Med & 2.430 & 2.430 & $12-19$ & 52,6 \\
\hline Ferranti et al. 21 & Estados Unidos & 2004 & Circulation & 1.960 & 1.960 & $15-19$ & ND \\
\hline Duncan et al. 17 & Estados Unidos & 2004 & Diabetes Care & 991 & 991 & $12-19$ & 53,9 \\
\hline Morán et al. 14 & México & 2004 & Diabetes Care & 965 & 965 & $10-18$ & 51,7 \\
\hline Goodman et al. 22 & Estados Unidos & 2004 & J Pediatr & 1.513 & 1.513 & $12-18$ & 50,0 \\
\hline Weiss et al. 23 & Estados Unidos & 2004 & N Engl J Med & 490 & 490 & $4-20$ & 46,9 \\
\hline Agirbasli et al. 19 & Turquia & 2006 & Metabolism & 1.385 & 1.385 & $10-17$ & 50,1 \\
\hline Atabek et al. 20 & Turquia & 2006 & Diabetes Res Clin Pract & 169 & 69 & $7-18$ & 44,9 \\
\hline Esmaillzadeh et al. 16 & Irã & 2006 & Obesity & 3.036 & 3.036 & $10-19$ & 53,4 \\
\hline Sanders 24 & Estados Unidos & 2006 & Ann Pharmacother & 52 & 52 & $12-20$ & 57,5 \\
\hline Fu et al. 12 & China & 2007 & Int J Obes & 348 & 348 & $7-16$ & 40,2 \\
\hline Kim et al. 13 & Coréia do Sul & 2007 & Diabetes Res Clin Pract & 2.165 & 2.165 & $12-19$ & 50,3 \\
\hline Sartorio et al. 26 & Itália & 2007 & Diabetes Res Clin Pract & 439 & 439 & $8-18$ & 56,4 \\
\hline Jolliffe \& Janssen 25 & Estados Unidos & 2007 & J Am Coll Cardiol & 6.002 & 6.002 & $12-19$ & 48,1 \\
\hline Singh et al. 15 & Índia & 2007 & Diabet Med & 1.083 & 1.083 & $12-17$ & 47,3 \\
\hline Castillo et al. 18 & México & 2007 & J Adolesc Health & 1.366 & 927 & $7-24$ & 56,6 \\
\hline
\end{tabular}

ND: informação não disponível no estudo.

* Abreviação dos periódicos de acordo com as normas de Vancouver. 
apresentam a prevalência de síndrome metabólica determinada pelos diferentes critérios. A menor prevalência de síndrome metabólica foi de 2,2\% (IC95\%: 1,5-3,1), entre adolescentes tur$\cos 19$, enquanto a maior prevalência encontrada foi entre adolescentes diabéticos (52,1\%; IC95\%: 47,4-56,9) 26. Todos os estudos observaram que os rapazes apresentaram prevalências da síndrome metabólica superiores às moças, e a diferença variou de 0,4 16 a dez pontos percentuais 19 . Dentre os estudos que apresentaram a prevalência da síndrome metabólica por faixa etária 9,15,18,21,25, os adolescentes mais novos (10-14 anos) apresentaram maiores freqüências da síndrome metabólica.

Dentre os componentes da síndrome, o triglicéride elevado apresentou as maiores prevalências, variando entre 4,9\% (IC95\%: 3,8-6,1) 22 e 75\% (IC95\%: 70,6-78,9) 26. A variação das prevalências dos demais componentes foi: baixo nível de HDL colesterol (8,1\%; IC95\%: 5,3-10,6 17 a 42,2; IC95\%: 41,0-44,6 16); obesidade central (4,9\%; IC95\%: 3,8-6,1 19 a 27,7\%; IC95\%: 24,8 $30,514)$; pressão arterial elevada (5,4\%; IC95\%: 3,3-8,3 12 a 23,8\%; IC95\%: 22,3-25,3 16) e níveis elevados de glicose (0,4\%; IC95\%: 0,0-1,4 18 a 7,7\%; IC95\%: 6,0-9,4 14). Como esperado nos estudos com obesos 12,20,23,26 e diabéticos tipo II 24, observa-se que os componentes da síndrome metabólica são mais freqüentes (Tabela 2).

A Tabela 2 demonstra ainda que cinco estudos investigaram a prevalência da síndrome metabólica pelos critérios da OMS e NCEP-ATP III. Contudo, apenas três destes estudos apresentam as prevalências por ambos os critérios. Morán et al. 14 encontraram valores superiores pelo critério do NCEP-ATP III que pelo critério da OMS. Todavia, no estudo realizado por Goodman et al. 22 , a prevalência foi maior conforme o critério da OMS (8,4\%; IC95\%: 7,0-9,9 contra

Descrição dos critérios diagnósticos da síndrome metabólica e das prevalências (\%) com intervalo de 95\% de confiança.

\begin{tabular}{|c|c|c|c|c|c|c|c|}
\hline Referência & Critério utilizado & $\begin{array}{l}\text { Obesidade } \\
\text { abdominal } \\
\text { [\% (IC } 95 \%)]\end{array}$ & $\begin{array}{c}\text { Glicose } \\
\text { elevada } \\
\text { [\% (IC95\%)] }\end{array}$ & $\begin{array}{c}\text { Triglicéride } \\
\text { elevado } \\
\text { [\% (IC95\%)] }\end{array}$ & $\begin{array}{c}\text { Baixo } \\
\text { HDL } \\
\text { colesterol } \\
\text { [\% (IC95\%)] }\end{array}$ & $\begin{array}{c}\text { Pressão } \\
\text { arterial } \\
\text { elevada } \\
\text { [\% (IC95\%)] }\end{array}$ & $\begin{array}{l}\text { Prevalência } \\
\text { da síndrome } \\
\text { metabólica } \\
\text { [\% (IC95\%)] }\end{array}$ \\
\hline Cook et al. 9 & NCEP-ATP III & $9,8(8,2-11,4)$ & $1,5(0,1-2,8)$ & $23,4(19,9-27,0)$ & $23,3(20,6-26,0)$ & $4,9(3,4-6,4)$ & $4,2(2,9-5,4)$ \\
\hline Ferranti et al. 21 & NCEP-ATP III & $21,0(19,2-22,8)$ & $1,0(0,6-1,5)$ & $9,5(8,2-10,8)$ & $42,2(39,9-44,4)$ & $3,1(2,4-3,9)$ & $9,2(7,9-10,5)$ \\
\hline Duncan et al. 17 & NCEP-ATP III & $11,8(8,6-15,1)$ & $7,6(4,8-10,4)$ & $23,2(18,6-27,9)$ & $8,1(5,3-10,6)$ & $23,4(19,3-27,6)$ & $6,4(3,8-8,9)$ \\
\hline Morán et al. 14 & $\begin{array}{c}\text { OMS e } \\
\text { NCEP-ATP III }\end{array}$ & $27,7(24,8-30,5)$ & $7,7(6,0-9,4)$ & $9,5(7,6-11,4)$ & $20,8(18,3-23,4)$ & $7,1(5,5-8,8)$ & $\begin{array}{l}4,5(3,2-5,8) \text { * } \\
6,5(4,7-7,8) \text { ** }\end{array}$ \\
\hline Goodman et al. 22 & $\begin{array}{c}\text { OMS e } \\
\text { NCEP-ATP III }\end{array}$ & $22,3(20,2-24,4) * \star$ & $1,7(1,1-2,5)$ ** & $4,9(3,8-6,1)$ ** & $\begin{array}{c}4,9(3,8-6,1) * \\
20,0(18,0-22,1) \text { ** }\end{array}$ & $12,3(10,7-14,1)$ ** & $\begin{array}{l}8,4(7,0-9,9) \text { * } \\
4,2(3,3-5,4) \text { ** }\end{array}$ \\
\hline Weiss et al. 23 & $\begin{array}{c}\text { OMS e } \\
\text { NCEP-ATP III }\end{array}$ & $58,0(53,4-62,3)$ ** & $27,0(23,1-31,1)$ ** & $17,0(13,7-20,8)$ ** & ND & $14,0(11,1-17,5)$ ** & $\begin{array}{c}38,7(34,4-43,2) \text { * } \\
27,0(23,0-31,1) \text { ** }\end{array}$ \\
\hline Agirbasli et al. 19 & NCEP-ATP III & $4,9(3,8-6,1)$ & $0,5(0,2-1,0)$ & $18,7(16,6-20,8)$ & $13,4(11,7-15,3)$ & $15,7(13,8-17,7)$ & $2,2(1,5-3,1)$ \\
\hline Atabek et al. 20 & OMS & ND & $14,4(7,1-25,0)$ & $44,9(32,9-57,4)$ & $20,2(11,6-31,6)$ & $31,8(21,2-44,2)$ & $37,6(26,3-50,1)$ \\
\hline Esmaillzadeh et al. 16 & NCEP-ATP III & $10,0(9,0-11,1)$ & $0,6(0,3-0,9)$ & $37,5(35,8-39,3)$ & $42,8(41,0-44,6)$ & $23,8(22,3-25,3)$ & $10,1(9,0-11,1)$ \\
\hline Sanders 24 & $\begin{array}{c}\text { OMS e } \\
\text { NCEP-ATP III }\end{array}$ & $92,5(81,4-97,8)$ * & $92,5(81,4-97,8)$ * & $75,0(61,1-85,9)$ * & $75,0(61,1-85,9)$ * & $87,5(76,6-95,6)$ * & $10,3(3,2-21,0)$ * \\
\hline Fu et al. 12 & $\begin{array}{c}\text { OMS e } \\
\text { NCEP-ATP III }\end{array}$ & $29,7(24,8-34,6)$ ** & ND & $13,8(10,3-17,8)$ ** & ND & $5,4(3,3-8,3)$ ** & $10,3(7,3-14,0)$ ** \\
\hline Kim et al. 13 & NCEP-ATP III & $25,1(23,2-26,9)$ & $6,8(5,7-7,9)$ & $30,5(28,5-32,4)$ & $25,6(23,7-27,4)$ & $19,5(17,8-21,2)$ & $9,2(8,0-10,5)$ \\
\hline Sartorio et al. 26 & NCEP-ATP III & ND & $92,5(89,6-94,7)$ & $75,0(70,6-78,9)$ & $75,0(70,6-78,9)$ & $90,0(86,8-92,6)$ & $52,1(47,4-56,9)$ \\
\hline Jolliffe \& Janssen 25 & NCEP-ATP III & ND & ND & ND & ND & ND & $7,6(6,9-8,3)$ \\
\hline Singh et al. 15 & NCEP-ATP III & $4,0(2,8-5,2)$ & $5,0(3,7-6,3)$ & $20,4(18,0-22,8)$ & $25,8(23,2-28,4)$ & $7,8(6,2-9,4)$ & $4,2(3,1-5,5)$ \\
\hline Castillo et al. 18 & NCEP-ATP III & $22,2(18,5-26,2)$ & $0,4(0,0-1,4)$ & $42,8(38,3-47,3)$ & $89,3(86,1-91,9)$ & $6,9(4,7-9,4)$ & $15,3(12,2-18,8)$ \\
\hline
\end{tabular}

NCEP-ATP III: National Cholesterol Education Program's Adult Treatment Panel IIl; OMS: Organização Mundial da Saúde; ND: informação não disponível no estudo.

* Prevalência de acordo com o critério da OMS;

** Prevalência de acordo com o critério do NCEP-ATP III. 
4,2\%; IC95\%: 3,3-5,4, utilizando o critério do NCEP-ATP III). Weiss et al. 23 também verificaram maior prevalência quando adotado o critério da OMS em adolescentes obesos.

\section{Discussão}

Este estudo teve como objetivo revisar a literatura acerca da prevalência de síndrome metabólica entre adolescentes. Os índices de prevalência entre os estudos incluídos variaram de $2,2 \%$ (IC95\%: 1,4-3,0) 19 a 52,1\% (IC95\%: 47,4-56,9) 26. Os estudos que utilizaram o critério do NCEPATP III apresentaram prevalências mais baixas; entre 2,2 (IC95\%: 1,4-3,0) 19 e 15,3 (IC95\%: 12,2$18,8) 18$ pontos percentuais, quando comparados aos estudos que utilizaram o critério da OMS. Entretanto, dentre as cinco pesquisas que utilizaram o critério da OMS, três foram conduzidas com obesos, fato que pode explicar os elevados índices de síndrome metabólica.

Um ponto importante a ser discutido são os critérios utilizados para definição da síndrome metabólica (NCEP-ATP III e OMS). As diferenças nos resultados podem ser, pelo menos parcialmente, explicadas por aspectos metodológicos. Por exemplo, a utilização de um critério não garante total comparabilidade entre os estudos, uma vez que os pontos de corte para os componentes da síndrome metabólica podem variar. Neste contexto, os estudos asiáticos 12,13 utilizaram os pontos de corte para obesidade abdominal estabelecidos pelos ministérios de comércio, indústria e energia daqueles países (Coréia do Sul e China), e a prevalência para este fator de risco foi de 29,7\% (IC95\%: 24,8-34,6) 12 e 25,1\% (IC95\%: 23,2-26,9) 13. Outros estudos utilizaram os pontos de corte propostos por Taylor et al. 10 e as prevalências variaram entre 4,0 (IC95\%: 2,8-5,2) 15 e 27,7\% (IC95\%: 24,8-30,5) 14. Apesar das diferenças metodológicas, tal fator de risco apresentou índices elevados, o que pode ser explicado pela tendência de aumento da adiposidade nesta idade 27 . Outro fator que pode influenciar não somente a prevalência da síndrome metabólica, mas também os fatores associados está relacionado à precisão das medidas. O efeito de erros de classificação diferencial (erro influenciado pelo status da doença ou exposição) ou não diferencial sobre a prevalência da síndrome metabólica é imprevisível, podendo superestimar ou subestimar a verdadeira prevalência 28 . No contexto do presente estudo, é plausível acreditar que a validade dos critérios diagnósticos e instrumentos utilizados variem de acordo com características dos adolescentes. Mas, independente dos critérios utilizados ou características dos adolescentes, os estudos foram consistentes em relação à prevalência da síndrome metabólica entre adolescentes: 13 dos 16 estudos revisados apresentaram prevalências da síndrome metabólica superiores a 5\%; 8 estudos apresentaram prevalências superiores a $10 \%$. Em relação às medidas de associação, o erro não diferencial tende a subestimá-las enquanto os erros diferenciais podem superestimar ou subestimar as associações 28 . Porém, ressalta-se que o presente estudo investigou a prevalência da síndrome metabólica e sugerese que futuros trabalhos considerem a avaliação dos fatores associados à síndrome metabólica em adolescentes.

Quando analisamos a prevalência dos fatores de risco para o desenvolvimento da síndrome individualmente, o perfil lipídio alterado (hipertrigliceridemia e baixo nível sérico de HDL-C) foram os componentes com maiores prevalências na maioria das pesquisas. Este resultado é consistente com outras pesquisas epidemiológicas, que demonstram que cerca de $65 \%$ dos adolescentes têm dislipidemia 29 . A dislipidemia tem forte associação com os valores pressóricos de adolescentes 30,31 . A proporção de adolescentes com pressão arterial elevada nos estudos revisados variou de moderada a alta (3,1; IC95\%: 2,4-3,9 21 a 23,8\% IC95\%: 22,3-25,3 17). O fator de risco com menor prevalência foi a hiperglicemia, que variou entre 0,4\% (IC95\%: 0,0-1,4) 18 a 14,4\% (IC95\%: 7,1-25,0) 20, excluindo-se o estudo realizado com adolescentes diabéticos tipo 2 26, que apresentou proporção de 92,5\% (IC95\%: 89,6-94,7), valores esperados visto que o diabetes é caracterizado por uma deficiência no metabolismo da glicose. Todavia o grupo de investigadores e estudos do diabetes verificou em jovens dos Estados Unidos um aumento elevado no número de casos de 17,0 para 49,4 a cada cem mil adolescentes 32 .

De acordo com o levantamento realizado, identificou-se que o Brasil carece de estudos que estimem a prevalência de síndrome metabólica. Tais estudos são importantes, já que estudos de tendência temporal sobre o excesso de peso entre adolescentes brasileiros apontam para aumentos na prevalência do excesso de peso 33,34 , e de acordo com os estudos mencionados, adolescentes obesos têm maior probabilidade de desenvolver a síndrome metabólica. Portanto, é provável que a ocorrência de síndrome metabólica entre brasileiros também esteja aumentando.

Conclui-se que, apesar das diferenças metodológicas e da falta de um consenso no diagnóstico da síndrome metabólica, os poucos estudos que avaliaram a prevalência da síndrome apontam para prevalências elevadas em ado- 
lescentes. Esta prevalência é substancialmente maior entre adolescentes obesos ou diabéticos. Há necessidade de estudos com adolescentes brasileiros, bem como estudos que avaliem a

\section{Resumo}

O objetivo do estudo foi revisar a literatura sobre a prevalência da síndrome metabólica e seus componentes em adolescentes (10-19 anos). Realizou-se busca nas bases eletrônicas MEDLINE e SciELO, referências dos artigos encontrados e contato com autores. Apenas artigos originais que utilizaram os critérios da Organização Mundial da Saúde (OMS) ou do National Cholesterol Education Program's Adult Treatment Panel III (NCEP-ATP III) foram considerados. Dezesseis estudos preencheram os critérios de inclusão. Destes, oito estudos foram realizados em países de renda baixa ou média, mas nenhum no Brasil. A prevalência de síndrome metabólica entre os estudos que utilizaram o critério da NCEP-ATP III variou de 4,2\% a 15,4\%. Utilizando-se o critério da OMS, as prevalências foram mais elevadas: $4,5 \%$ a $38,7 \%$. Dentre os componentes da síndrome, o triglicéride elevado apresentou maior prevalência, com valores entre 4,9\% a 75\%, e a glicose elevada apresentou as menores prevalências. Concluise que, apesar do baixo número de estudos localizados, a ocorrência da síndrome metabólica entre adolescentes é um problema de saúde com alta prevalência. A prevalência de síndrome metabólica em adolescentes brasileiros é desconhecida.

Adolescente; Síndrome Metabólica X; Obesidade efetividade de intervenções no estilo de vida (ou seja, hábitos alimentares e práticas de atividade física, por exemplo) sobre a prevalência e incidência da síndrome metabólica.

\section{Colaboradores}

A. C. F. Moraes e C. S. Fulaz participaram da estruturação, elaboração do artigo e busca das referências utilizadas no manuscrito. E. R. Netto-Oliveira e F. F. Reichert participaram da elaboração, análise e apresentação dos resultados e análise crítica do artigo. 


\section{Referências}

1. Cook S. The metabolic syndrome: antecedent of adult cardiovascular disease in pediatrics. J Pediatr 2004; 145:427-30.

2. Lopes HF. Hipertensão arterial e síndrome metabólica: além da associação. Rev Soc Cardiol Estado de São Paulo 2003; 13:64-77.

3. Organização Mundial da Saúde. Obesidade: prevenindo e controlando a epidemia global: relatório da consultoria da OMS. São Paulo: Editora Roca; 2004.

4. Pazin J, Frainer DES. Obesity and motor development - a cross-sectional study with Brazilians school children. FIEP Bulletin 2007; 77:453-6.

5. Alberti FGMM, Zimmet PZ. Definition, diagnosis and classification of diabetes mellitus and its complications. Part 1: diagnosis and classification of diabetes mellitus, provisional report of a WHO consultation. Diabet Med 1998; 15:539-53.

6. Expert Panel on Detection, Evaluation and Treatment of High Blood Cholesterol in Adults. Executive summary of the Third Report of the National Cholesterol Education Program (NCEP) Expert Panel on Detection, Evaluation and Treatment of High Cholesterol. JAMA 2001; 285:2486-97.

7. Sociedade Brasileira de Cardiologia. I diretriz brasileira de diagnóstico e tratamento da síndrome metabólica. Arq Bras Cardiol 2005; 84:1-28.

8. Lottenberg AS, Glezer A, Turatti LA. Metabolic syndrome: identifying the risk factors. J Pediatr 2007; 83 Suppl:S204-8.

9. Cook S, Weitzman M, Auinger P, Nguyen M, Dietz WH. Prevalence of a metabolic syndrome phenotype in adolescents: findings from the third National Health and Nutrition Examination Survey, 1988-1994. Arch Pediatr Adolesc Med 2003; 157:821-7.

10. Taylor RW, Jones IE, Williams SM, Goulding A. Evaluation or waist circumference, waist-to-hip ratio, and the conicity index as screening tools for high trunk fat mass, as measured by dual-energy $\mathrm{X}$-ray absorptiometry, in children aged 3-19 years. Am J Clin Nutr 2000; 72:490-5.

11. Centers for Disease Control and Prevention. Growth charts for the United States 2000: methods and development. http://www.cdc.gov/ growthcharts (acessado em 05/Out/2007).

12. Fu JF, Liang L, Zou CC, Hong F, Wang CL, Wang $\mathrm{XM}$, et al. Prevalence of the metabolic syndrome in Zhejiang Chinese obese children and adolescents and the effect of metformin combined with lifestyle intervention. Int J Obes 2007; 31:15-22.

13. Kim HM, Park J, Kim HS, Kim DH. Prevalence of the metabolic syndrome in Korean adolescents aged 12-19 years from the Korean National Health and Nutrition Examination Survey 1998 and 2001. Diabetes Res Clin Pract 2007; 75:111-4.

14. Morán MR, Vázquez BS, Violante R, Romero FG. Metabolic syndrome among children and adolescents aged 10-18 years. Diabetes Care 2004; 27:2516-7.

15. Singh R, Bhansali A, Sialy R, Aggarwal A. Prevalence of metabolic syndrome in adolescents from north Indian population. Diabet Med 2007; 24:195-9.
16. Esmaillzadeh A, Mirmiran P, Azadbakht L, Etemadi A, Azizi F. High prevalence of the metabolic syndrome in Iranian adolescents. Obesity 2006; 14:377-82.

17. Duncan GE, Li SM, Zhou XH. Prevalence and trends of a metabolic syndrome phenotype among U.S. adolescents, 1999-2000. Diabetes Care 2004; 27:2438-43.

18. Castillo EH, Borges G, Talavera JO, Orozco R, Vargas-Aleman $\mathrm{C}$, Huiltrón-Bravo, et al. Body mass index and the prevalence of metabolic syndrome among children and adolescents in two Mexican populations. J Adolesc Health 2007; 40:521-6.

19. Agirbasli M, Cakirb S, Ozmeb S, Cilivc G. Metabolic syndrome in Turkish children and adolescents. Metabolism 2006; 55:1002-6.

20. Atabek ME, Pirgon O, Kurtoglu S. Prevalence of metabolic syndrome in obese Turkish children and adolescents. Diabetes Res Clin Pract 2006; 72:315-21.

21. Ferranti SD, Gauvreau K, Ludwig DS, Neufeld EJ, Newburger JW, Rifai N. Prevalence of the metabolic syndrome in American adolescents: findings from the Third National Health and Nutrition Examination Survey. Circulation 2004; 110:2494-7.

22. Goodman E, Daniels SR, Morrison JA, Huang B, Dolan LM. Contrasting prevalence of and demographic disparities in the World Health Organization and National Cholesterol Education Program Adult Treatment Panel III definitions of metabolic syndrome among adolescents. J Pediatr 2004; 145:445-51.

23. Weiss R, Dziura J, Burgert TS, Tamborlane WV, Taksali SE, Yeckel CW, et al. Obesity and the metabolic syndrome in children and adolescents. N Engl J Med 2004; 350:2362-74.

24. Sanders BH. Prevalence and treatment of metabolic syndrome in adolescents with type 2 diabetes. Ann Pharmacother 2006; 9:1517-21.

25. Jolliffe CJ, Janssen I. Development of age-specific adolescent metabolic syndrome criteria that are linked to the adult treatment panel III and International Diabetes Federation criteria. J Am Coll Cardiol 2007; 49:891-8.

26. Sartorio A, Agosti F, De Col A, Mornati D, Francescato MP. Prevalence of the metabolic syndrome in Caucasian obese children and adolescents: comparison between three different definition criteria. Diabetes Res Clin Pract 2007; 77:341-2.

27. Freedman DS, Srinivasan SR, Valdez RA, Williamson DF, Berenson GS. Secular increases in relative weight and adiposity among children over two decades: the Bogalusa Heart Study. Pediatrics 1997; 99:420-6.

28. Mertens TE. Estimating the effects of misclassification. Lancet 1993; 342:418-21.

29. Carvalho DF, Paiva AA, Melo ASO, Ramos AT, Medeiros JS, Medeiros CCM, et al. Perfil lipídico e estado nutricional de adolescentes. Rev Bras Epidemiol 2007; 10:491-8. 
30. Freedman DS, Kahn HS, Mei Z, Grummer-Strawn LM, Dietz WH, Srinivasan SR, et al. Relation of body mass index and waist-to-height ratio to cardiovascular disease risk factors in children and adolescents: the Bogalusa Heart Study. Am J Clin Nutr 2007; 86:33-40.

31. Lee S, Bacha F, Arslanian AS. Waist circumference, blood pressure and lipids components of the metabolic syndrome. J Pediatr 2006; 149:809-16.

32. Diabetes in Youth Study Group. Incidence of diabetes in youth in the United States. JAMA 2007; 297:2716-24
33. Wang Y, Monteiro C, Popkin BM. Trends of obesity and underweight in older children and adolescents in the United States, Brazil, China, and Russia. Am J Clin Nutr 2002; 75:971-7.

34. Ministério da Saúde/Instituto Brasileiro de Geografia e Estatística. Pesquisa de orçamentos familiares 2002-2003. Antropometria e análise do estado nutricional de crianças e adolescentes no Brasil, 2006. http://www.saude.gov.br (acessado em 20/Mar/2008).

Recebido em 19/Ago/2008

Versão final reapresentada em 27/Nov/2008

Aprovado em 17/Dez/2008 